\title{
Functions of Fibroblast Growth Factor (FGF)-2 and FGF-5 in Astroglial Differentiation and Blood-Brain Barrier Permeability: Evidence from Mouse Mutants
}

\author{
Bernhard Reuss, ${ }^{1}$ Rosanna Dono, ${ }^{2}$ and Klaus Unsicker ${ }^{1}$ \\ ${ }^{1}$ Department of Neuroanatomy, IZN, University of Heidelberg, 69120 Heidelberg, Germany, and ${ }^{2}$ Department of Developmental Biology, University of \\ Utrecht, 3584 CH Utrecht, The Netherlands
}

\begin{abstract}
Multiple evidence suggests that fibroblast growth factors (FGFs), most prominently FGF-2, affect astroglial proliferation, maturation, and transition to a reactive phenotype in vitro, and after exogenous administration, in vivo. Whether this reflects a physiological role of endogenous FGF is unknown. Using FGF-2 and FGF-5 single- and double mutant mice we show now a region-specific reduction of glial fibrillary acidic protein (GFAP), but not of S100 in gray matter astrocytes. FGF-2 is apparently the major regulator of GFAP, because in mice deficient for FGF-2, GFAP is distinctly reduced in cortex and striatum, whereas in FGF- $5^{-1-}$ animals only a reduction in the midbrain tegmentum can be observed. In FGF- $2^{-l-} / \mathrm{FGF}-5^{-1-}$ double mutant animals, GFAP-immunoreactivity is reduced in all three brain regions. Cortical astrocytes cultured from FGF- $2^{-1-} / \mathrm{FGF}-5^{-1-}$ double mutant mice revealed reduced levels of GFAP, but not S100 as compared with wild-type littermates. This phenotype could be rescued by exogenous FGF-2 but not FGF-5 (10 ng/ml). Electron microscopy revealed reduced levels of intermediate filaments in perivascular astroglial endfeet. This defect was accompanied by enhanced permeability of the blood-brain barrier (BBB), as detected by albumin extravasation. Levels of the tight junction proteins Occludin and ZO-1 were reduced in blood vessels of FGF-2 ${ }^{-1-} / \mathrm{FGF}-5^{-1-}$ double mutant mice as compared with wild-type littermates. Our data support the notion that endogenous FGF-2 and FGF-5 regulate GFAP expression in a region-specific manner. The observed defect in astroglial differentiation is accompanied by a defect in BBB function arguing for an indirect or direct role of FGFs in the regulation of BBB permeability in vivo.
\end{abstract}

Key words: astrocyte; FGF; GFAP; BBB; tight junctions; occludin

\section{Introduction}

During their whole life span, cells in the CNS are subject to plastic changes that depend, in part, on actions of protein growth factors, as e.g., fibroblast growth factors (FGFs). Spatial distribution patterns of FGFs, in conjunction with their effects in vitro and in vivo, indicate multiple roles of FGFs during CNS development and subsequent to lesions (for review, see Bieger and Unsicker, 1996). A major target for actions of FGF are astrocytes that are critical for the maintenance of neural homeostasis and for neuronal regeneration after brain damage (for review, see Ransom and Kettenmann, 1995). Because astrocytes synthesize FGF-2 (Gomez-Pinilla et al., 1994) and express FGF receptors (FGFRs) -2 and -3 (Reuss et al., 2000a), FGF-2 is a major candidate for the autocrine and/or paracrine regulation of astroglial cell differen-

Received Dec. 27, 2002; revised May 2, 2003; accepted May 8, 2003.

This work was supported by Deutsche Forschungsgemeinschaft Grants Re1381/2-1 and Re1381/5-1 and from the Medical Faculty of the University of Heidelberg (Friedrich-Fischer-Nachlass). We thank Gail Martin (Anatomy and Developmental Biology Program, University of California at San Francisco) for providing us with FGF- $2^{-/} / \mathrm{FGF}-5^{-/-}$ double mutant mice. We also thank Barbara Brühl, Elisabeth Specht-Delius, Jutta Fey, and Petra Christmann for excellent technical support.

Correspondence should be addressed to Dr. Bernhard Reuss, Neuroanatomy, IZN, University of Heidelberg, Im Neuenheimer Feld 307, 69120 Heidelberg, Germany. E-mail: bernhard.reuss@urz.uni-heidelberg.de.

Copyright $\odot 2003$ Society for Neuroscience $\quad$ 0270-6474/03/236404-09\$15.00/0 tiation, functions, and transition to a "reactive" phenotype seen after lesions (Frautschy et al., 1991; Reuss et al., 1998, 2000a,b).

FGF-5 is another brain resident FGF, regulating neuron differentiation and survival (Lindholm et al., 1994), as well as astroglial properties in vitro (Reuss et al., 2000a). FGF-5 is expressed in most brain regions, especially in frontal cortex, hippocampus, and hypothalamus (Haub et al., 1990; Gomez-Pinilla and Cotman, 1993). FGF-5 is located in neurons, probably affecting astrocytes via paracrine actions (Gomez-Pinilla and Cotman, 1993). As previously shown in vitro, FGF-5 suppresses astroglial gap junctions in primary mesencephalic astroglial cultures (Reuss et al., 2000a). As for FGF-2, most data concerning FGF-5 effects on astrocytes are derived from cell culture studies, leaving the question open whether effects of FGF-5 on astroglial cells reflect a real physiological function.

A specific marker for astroglial cell differentiation is the intermediate filament component glial fibrillary acidic protein (GFAP), which determines mechanical stability of astrocytes (Eng et al., 1971; Bignami et al., 1972; Eng, 1985). Astrocytes are tightly associated with brain capillaries and, by inducing endothelial tight junctions, contribute to the formation of the bloodbrain barrier (BBB) (Prat et al., 2001). Because mechanical properties of astrocytes influence proper functioning of the BBB (Pekny et al., 1998), FGF-dependent alterations in astroglial 
GFAP expression could be a cause for pathological changes to BBB permeability.

To clarify the physiological roles of endogenous FGF-2 and FGF-5 in regulating astroglial cell differentiation and BBB permeability, we studied astrocytes in different brain regions of FGF$2^{-1-}$ and FGF- $5^{-1-}$ single- and in FGF- $2^{-1-} / \mathrm{FGF}-5^{-1-}$ double mutant mice focusing on the expression of GFAP and S100 studied by immunocytochemistry, Western blot, RT-PCR, and electron microscopy. BBB permeability was analyzed by immunochemical detection of albumin extravasation and expression of the endothelial tight junction proteins ZO-1 and Occludin. Our results show for the first time that endogenous FGF-2, and to a lesser extent FGF-5, are important determinants of astroglial cell differentiation in vivo and that FGF-dependent astroglial regulation has a major impact on the integrity of the BBB.

\section{Materials and Methods}

Animals. FGF-2 ${ }^{-1-}$ single knock-out $(\mathrm{KO})$ mice used for the present study were of the strain generated by Rosanna Dono and Rolf Zeller (Dono et al., 1998). FGF-5 $5^{-1-}$ single KO mice (Hébert et al., 1994) were obtained from Jackson Laboratories (Bar Harbor, ME). FGF-2 ${ }^{-1-}$ /FGF$5^{-1-}$ double-deficient mice were generated by crossing an FGF-2deficient strain of mice (Zhou et al., 1998) with a strain bearing a defect for FGF-5 (Hébert et al., 1994). Wild-type littermates were used as control animals.

Primary astroglial cultures. Cultures of astroglial cells from the cortices of newborn mice were prepared as described previously (Reuss et al., 1998). In brief, tissue samples were freed from meninges and collected in HBSS (Invitrogen, Karlsruhe, Germany). After trituration with a firepolished Pasteur pipette and centrifugation, the pellet was resuspended in DMEM (Invitrogen) supplemented with $10 \%$ horse serum (Invitrogen) and penicillin (50 IU/ml)-streptomycin $(50 \mu \mathrm{g} / \mathrm{ml})$-neomycin (Invitrogen). The cell suspension resulting from six brains was seeded on 75 $\mathrm{cm}^{2}$ poly-L-lysine-coated tissue culture flasks (Corning Costar, Bodenheim, Germany). Cells were grown to confluency in DMEM with the abovementioned supplements, changing the culture medium every 2 or $3 \mathrm{~d}$. O2A-precursor and microglial cells were removed by shaking them off. Cells were split by trypsinization and seeded onto plastic cell culture dishes (60 mm in diameter; Becton Dickinson, Heidelberg, Germany) for Western blot and RT-PCR, or $12 \mathrm{~mm}$ coverslips in 24 well cell culture plates (Becton Dickinson) for immunocytochemistry as described below.

Antibodies. Immunochemical detection of the various antigens investigated in the present study was performed using the following antibodies. GFAP: mouse monoclonal (Sigma, Deisenhofen, Germany); S100: mouse polyclonal (Sigma); albumin: rabbit polyclonal (ICN Biomedicals, Eschwege, Germany); NeuN: mouse monoclonal (Chemicon, Temecula, CA); TH: mouse monoclonal (Roche, Basel, Switzerland); ZO-1: rat polyclonal (Chemicon); occludin: rabbit polyclonal (Zymed, San Francisco, CA); $\beta$-Actin: mouse monoclonal (Sigma).

Immunocytochemistry. For immunocytochemical detection, $10 \mu \mathrm{m}$ cryostat sections of freshly frozen brain tissue samples were mounted on poly-L-lysine-coated coverslips. Alternatively, primary astroglial cells cultured on $12 \mathrm{~mm}$ glass coverslips coated with poly-L-lysine were used. Sections or cultured cells were fixed for $10 \mathrm{~min}$ with $4 \%$ paraformaldehyde at room temperature, followed by a $10 \mathrm{~min}$ permeabilization with $0.1 \%$ Triton X-100 in PBS. After three times washing for 10 min with PBS, primary antibodies were applied to the coverslips at a dilution of 1:100 followed by a $2 \mathrm{hr}$ incubation at room temperature. Subsequently, sections were washed three times for 15 min with PBS and were then incubated with the appropriate FITC- or tetramethylrhodamine isothiocyanate-conjugated secondary antibodies (Dako, Glostrup, Denmark) at a dilution of 1:200 for $2 \mathrm{hr}$ at room temperature. After three washing steps with PBS for 15 min, coverslips were mounted with Fluorsave mounting medium (Calbiochem, Darmstadt, Germany) and were viewed with a Zeiss Axiophot photomicroscope equipped with epifluorescence (Carl Zeiss, Jena, Germany).

Quantifications. Numbers of S100-immunoreactive cells in a rectangle with a side length of $500 \mu \mathrm{m}$ were counted on seven consecutive sections (section interval, $50 \mu \mathrm{m}$ ) in six animals for each value. Albumin extravasation was quantified by counting blood vessels with or without apparent albumin extravasation in a rectangle with $500 \mu \mathrm{m}$ side length on seven consecutive sections (section interval, $50 \mu \mathrm{m}$ ) in six animals for each value. Vessels surrounded by albumin immunoreactivity at a distance exceeding one vessel diameter were counted. Rates are expressed as percentage of blood vessels showing albumin extravasation.

Western blotting. For immunoquantification, tissue samples were homogenized in electrophoresis sample buffer (Laemmli, 1970), and protein contents were determined using a densitometric method described by Henkel and Bieger (1994). Five micrograms of total cell protein per lane were separated by SDS-PAGE (Laemmli, 1970) and then transferred onto nitrocellulose membranes (Towbin et al., 1979). After transfer, the nitrocellulose membranes (Hybond-ECL; Amersham, Freiburg, Germany) were blocked with 3\% skim milk powder and $0.1 \%$ BSA in Trisbuffered saline (TBS; $150 \mathrm{mmol} / \mathrm{l}$ of $\mathrm{NaCl}, 10 \mathrm{mmol} / \mathrm{l}$ of Tris, $\mathrm{pH} 7.3$ ), then incubated overnight with primary antibodies as described above (diluted 1:2000 in TBS/1\% BSA), followed by a peroxidase-conjugated secondary antibody, which was detected by a chemiluminescent substrate system (ECL, Amersham, Freiburg, Germany). As a loadingcontrol, blots were stripped and reprobed with a polyclonal antibody directed to rat $\beta$-actin (mouse monoclonal; Sigma).

Isolation of total cellular RNA and RT-PCR. Total cellular RNA was isolated by Roti-Quick (Roth, Karlsruhe, Germany), according to the manufacturer's instructions, and quantified spectrophotometrically. Two micrograms of each sample was reverse-transcribed by adding $4 \mu \mathrm{l}$ of $5 \times \mathrm{RT}$ buffer $(50 \mathrm{mmol} / \mathrm{l}$ of Tris- $\mathrm{HCl}, \mathrm{pH} 8.3,50 \mathrm{mmol} / \mathrm{l}$ of $\mathrm{KCl}, 4$ $\mathrm{mmol} / \mathrm{l}$ of $\mathrm{MgCl}_{2}$, and $10 \mathrm{mmol} / \mathrm{l}$ of DTT; MBI Fermentas, St. Leon-Rot, Germany), $1 \mathrm{mmol} / \mathrm{l}$ of desoxy-nucleotide-tris-phosphate (dNTP) (Invitrogen), $50 \mathrm{ng} / \mu \mathrm{l}$ random hexanucleotide primers (Sigma, Deisenhofen, Germany), $0.875 \mathrm{U} / \mu \mathrm{l}$ RNase-Inhibitor (MBI Fermentas), and 20 $\mathrm{U} / \mu \mathrm{l}$ Moloney murine leukemia virus reverse transcriptase (MBI Fermentas), and water up to a final volume of $20 \mu$ l. Samples were incubated for $60 \mathrm{~min}$ at $37^{\circ} \mathrm{C}$ and for $5 \mathrm{~min}$ at $96^{\circ} \mathrm{C}$ to stop the reaction. Negative controls were performed by omitting the RNA in the reaction mix. PCR amplifications were performed in a GeneAmp PCR system 9600 thermal cycler (Perkin-Elmer, Boston, MA) in $0.2 \mathrm{ml}$ thin-walled reaction tubes using a hot-start method. To $2 \mu \mathrm{l}$ of cDNA template, $1 \times$ PCR buffer $(20$ $\mathrm{mmol} / \mathrm{l}$ of Tris- $\mathrm{HCl}, \mathrm{pH} 8.4 ; 50 \mathrm{mmol} / \mathrm{l}$ of $\mathrm{KCl}$ ), $3 \mathrm{mmol} / \mathrm{l}$ of $\mathrm{MgCl}_{2}, 0.4$ $\mathrm{mmol} / \mathrm{l}$ of dNTP, $1.25 \mu \mathrm{l}$ of TaqDNA polymerase (5 U/ $\mu \mathrm{l}$; Invitrogen), and $10 \mathrm{ng} / \mu \mathrm{l}$ of each forward and reverse primers were added to a final volume of $25 \mu$ l. Samples were denatured for $30 \mathrm{sec}$ at $94^{\circ} \mathrm{C}$, annealed at $60^{\circ} \mathrm{C}$ for $30 \mathrm{sec}$, and extended at $72^{\circ} \mathrm{C}$ for $45 \mathrm{sec}$. The following primer pairs were used for amplification: GFAP forward-primer: 5' GAA CAA CCT GGC TGC GTA TAG 3' reverse-primer: 5' GGT CTG CAA ACT TAG ACC GAT AC 3' (product length 292 bp); glyceraldehyde-3phosphate dehydrogenase (GAPDH) forward-primer: CAG AAC ATC ATC CCT GCA TCC ACT 3' reverse-primer: GTT GCT GTT GAA GTC ACA GGA GAC 3' (product length $284 \mathrm{bp}$ ). Twelve microliters of each sample was electrophoresed on $2 \%$ agarose gels in Tris acetate EDTA (Invitrogen) followed by ethidium bromide detection. Images were captured by a computer-assisted gel documentation system (INTAS, Göttingen, Germany).

Transmission electron microscopy. Animals were fixed by transcardial perfusion with $100 \mathrm{ml}$ of rinsing solution [0.5\% procaine-hydrochloride, $2.5 \%$ polyvinyl-pyrrolidone (molecular weight, 40, 000; PVP-40), and $0.025 \%$ heparin in $0.9 \% \mathrm{NaCl}$, adjusted to $\mathrm{pH} 7.3$ ], followed immediately by $100 \mathrm{ml}$ of fixation solution (1.5\% paraformaldehyde, $1.5 \%$ glutaraldehyde, and 2.5\% PVP-40, in $0.09 \mathrm{~mol} / \mathrm{l} \mathrm{Na-phosphate} \mathrm{buffer).} \mathrm{Brains}$ were dissected and removed from the skull, followed by submersion in fixation solution at $4^{\circ} \mathrm{C}$ overnight. Tissue blocks from the striatum were thoroughly rinsed $(5 \times)$ in cacodylate buffer, $\mathrm{pH} 7.2$, and osmicated in a mixture of $1.5 \%$ potassium-ferricyanide and $1 \%$ osmium-tetroxide in distilled water. The blocks were then rinsed several times in cacodylate buffer, $\mathrm{pH} 7.2$, followed by several rinsings in maleate buffer, $\mathrm{pH}$ 6.0. Blockstaining was performed in $1 \%$ uranyl acetate in the same buffer. All postfixation steps were done at room temperature. After final rinses in maleate buffer, the tissue was dehydrated in graded series of cold ethanol 

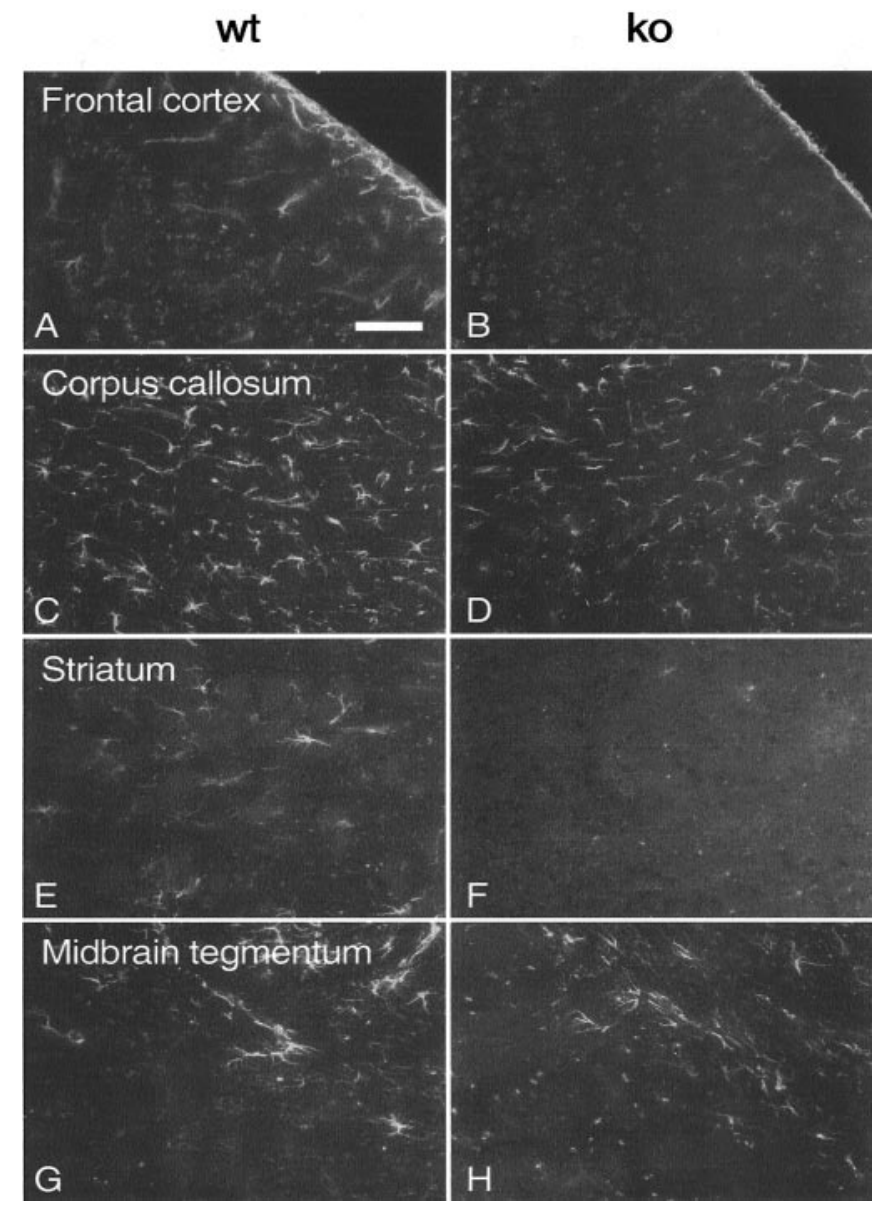

Figure 1. Protein expression of GFAP in different brain regions of mice deficient for FGF-2, as revealed by indirect fluorescence immunocytochemistry. $A$, Abundant GFAP-immunoreactive astrocytes can be observed in the frontal cortex of wild-type mice. $B$, In the same region of FGF-2 $2^{-/-}$animals, GFAP immunoreactivity has nearly disappeared. C, GFAP immunoreactivity is also abundant in the corpus callosum of wild-type mice, but in the knock-out (D) there is no overt reduction $(D)$. E, GFAP immunoreactivity in astrocytes of the striatum in wild-type mice is distinctly reduced in $\mathrm{FGF}-2^{-1-}$-deficient mice $(F)$. No overt differences in GFAP immunoreactivity between wild-type and FGF-2/FGF- 5 double mutant mice can be observed in the midbrain tegmentum $(G, H)$. Scale bar, $40 \mu \mathrm{m}$.

solutions ending with several rinses in $100 \%$ ethanol and embedded in Epon (Luft, 1961). Ultrathin sections (50-60 nm) were cut with an Ultrotome-III (LKB, Stockholm, Sweden) followed by staining with uranyl-acetate and lead-citrate (Reynolds, 1963) and examination with a Zeiss (Jena, Germany) EM-10 electron microscope. Images were taken from 100 striatal capillaries per animal of five wild-type and five FGF-2/ FGF-5 double mutant mice. Astroglial endfeet were categorized (five categories) according to the densities of intermediate filaments and mean values $( \pm \mathrm{SD})$ were plotted as percent of all vessels counted.

\section{Results}

GFAP immunoreactivity is region-specifically reduced in cortical, striatal, and mesencephalic gray, but not white matter astrocytes of FGF- $2^{-1-}$ and FGF-5 $5^{-1-}$ mice

FGF-2 is an established regulator of astroglial cell differentiation and GFAP expression in vitro (Perraud et al., 1988, 1990; Eclancher et al., 1990, 1996). To clarify whether this also applies to the in vivo situation, we analyzed the expression of GFAP in different brain regions of FGF-2 ${ }^{-1-}$-mice in comparison with wild-type littermates using immunofluorescence. Figure $1 \mathrm{~A}$ demonstrates abundant GFAP-positive astrocytes in the frontal cortex of FGF-2 ${ }^{+/+}$mice, whereas corresponding sections from

\section{FGF-5}

wt

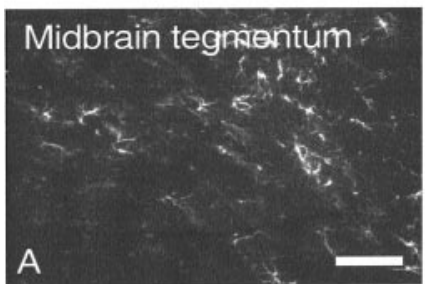

A

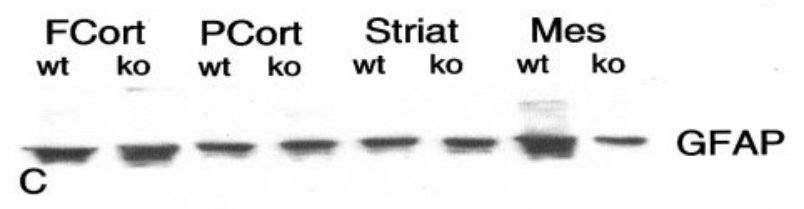

D

Figure 2. Immunohistochemical detection of GFAP in the midbrain tegmentum and Western blot analysis of GFAP in different brain regions of FGF- $5^{-1-}$ mice. $A$, Abundant GFAPimmunoreactive astrocytes can be observed in the midbrain tegmentum of wild-type mice. $B$, GFAP immunoreactivity in the same brain region of FGF- $5^{-1-}$ mice has largely disappeared. $C$, Western blot. In FGF- $5^{-1-}$ mice, immunoreactivity for GFAP is only reduced in the mesencephalon (Mes), whereas in frontal cortex (FCort), parietal cortex (PCort), and striatum (Striat) of FGF- $5^{-1-}$-deficient mice (ko), no apparent alterations as compared with material from wildtype littermates (wt) can be observed. Scale bar, $40 \mu \mathrm{m}$.

FGF- $2^{-1-}$-animals revealed hardly any GFAP-positive labeling. In contrast, comparisons of the corpus callosum from wild-type (Fig. 1C) and FGF-2 ${ }^{-/-}$animals (Fig. 1D) revealed no difference in GFAP immunostaining. In the striatum of FGF-2 $2^{-/-}$mice (Fig. $1 F$ ), immunoreactivity for GFAP was clearly reduced, as compared with wild-type littermates (Fig. $1 E$ ). Astrocytes in the midbrain tegmentum (Fig. $1 G, H$ ) of FGF- $2^{+/+}$and FGF-2 ${ }^{-1-}$ mice did not overtly differ with respect to GFAP immunoreactivity.

FGF-5 is another member of the FGF-family of growth factors, expressed in the mouse brain (Haub et al., 1990; GomezPinilla and Cotman, 1993; Ozawa et al., 1996). Because previous in vitro studies had revealed specific effects of exogenously applied FGF-5 related to gap junction coupling on cultured mesencephalic astrocytes (Reuss et al., 2000a), we next addressed a potential role of endogenous FGF-5 in the regulation of GFAP in the midbrain tegmentum. Figure 2, $A$ and $B$, shows that GFAP immunoreactivity in midbrain astroglial cells of FGF- $5^{-1-}$ mice is clearly reduced as compared with wild-type littermates. Western blot analyses (Fig. 2C,D) supported the notion that GFAP immunoreactivity is specifically reduced in the midbrain tegmentum, but not in frontal cortex, parietal cortex, and striatum.

\section{GFAP immunoreactivity and mRNA are reduced in gray} matter astrocytes of cortex, striatum, and midbrain tegmentum of FGF-2 $2^{-/-} / \mathrm{FGF}^{-1-} 5^{-/}$double mutant mice To address the issue of a putative synergism of FGF-2 and FGF-5 in the regulation of astroglial GFAP, we next analyzed FGF-2 ${ }^{-1-} / \mathrm{FGF}-5^{-1-}$ double mutant mice. Sections of the frontal cortex of double mutant mice (Fig. $3 B$ ) revealed a similar loss of GFAP immunoreactivity as seen in FGF-2 single mutants (compare Fig. $1 B$ ), corroborating the notion based on the analysis of FGF-5 knock-out mice that FGF-5 does not overtly contribute to the regulation of GFAP in cortical gray matter astro- 


\section{FGF-2/FGF-5}
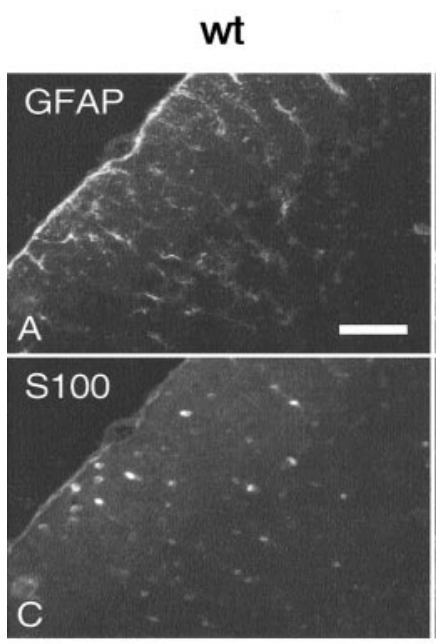

B
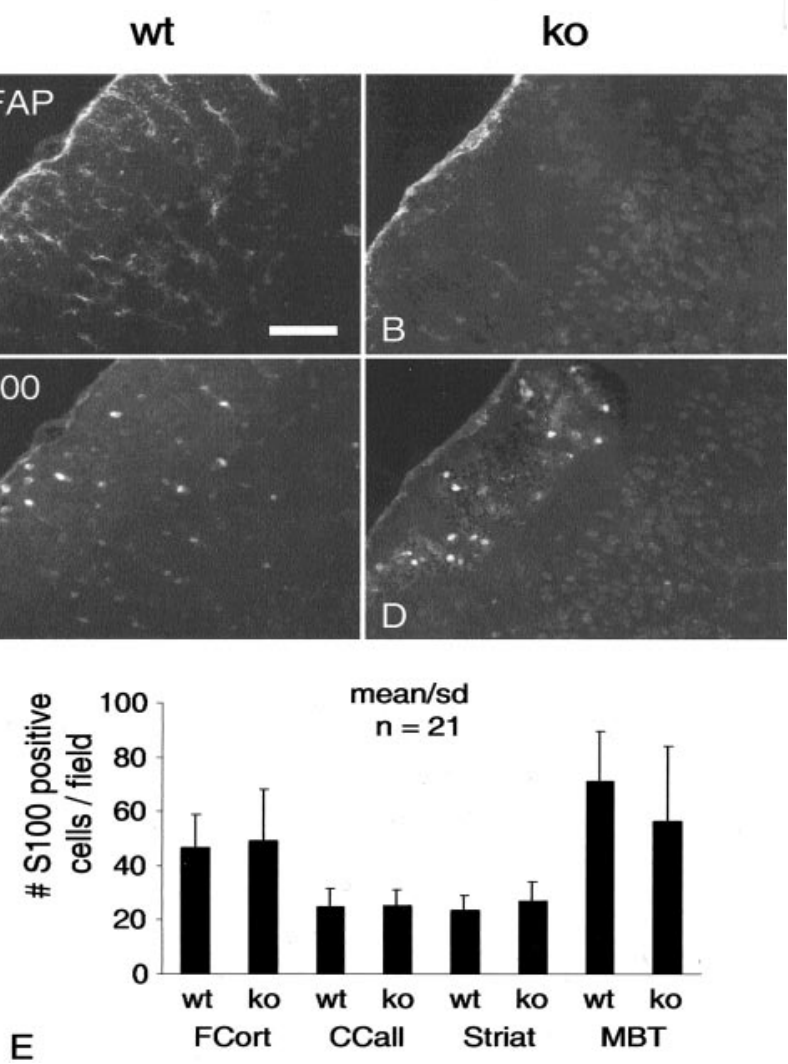

Figure 3. Immunocytochemical double staining for the astroglial marker proteins GFAP $(A$, $B)$ and $S 100(C, D)$ in the frontal cortex of FGF- $2^{-1-} / \mathrm{FGF}-5^{-1-}$ double mutant mice $(B, D)$ and wild-type littermates $(A, C)$. Note the apparent decrease of GFAP immunoreactivity in the frontal cortex of FGF- $2^{-1-} /$ FGF- $5^{-1-}$ double mutant mice, whereas $S 100$ levels are unchanged. E, Statistical evaluation of numbers of S100-positive cells in a given area $(500 \times 500 \mu \mathrm{m})$ in frontal cortex (FCort), corpus callosum (CCall), striatum (Striat), and midbrain tegmentum (MBT) of FGF-2 $2^{-l-} / \mathrm{FGF}-5^{-/-}$double mutant mice (ko) and their wild-type littermates (wt). Seven consecutive sections with a section interval of $50 \mu \mathrm{m}$ were evaluated for each of six wild-type and knock-out animals. Scale bar, $40 \mu \mathrm{m}$.

cytes of double mutant mice. The GFAP phenotype of the corpus callosum from $\mathrm{FGF}-2^{-1-} / \mathrm{FGF}-5^{-1-}$ double mutant mice was indistinguishable from that seen in FGF-2 and FGF-5 single mutants (data not shown, compare Fig. $1 C, D$ ), suggesting that FGF-2 and FGF-5 do not cooperate synergistically in the regulation of GFAP in this brain region in vivo. In the striatum, GFAP immunoreactivity was distinctly reduced in the double mutant mice as previously documented for FGF-2 ${ }^{-1-}$ mice (data not shown, compare Fig. $1 E, F$ ), whereas the reduction in GFAP immunoreactivity seen in mesencephalic astrocytes of FGF- $2^{-1-} /$ FGF-5 ${ }^{-1-}$ double mutants was indistinguishable from that shown for FGF- $5^{-1-}$ mice (data not shown, compare Fig. $2 A, B$ ). Together, these data suggest that FGF-2 regulates specifically GFAP immunoreactivity in cortical and striatal astrocytes, whereas FGF-5 serves this role in the midbrain tegmentum. However, FGF-2 and FGF-5 do not appear to act synergistically in either of the analyzed brain regions.

The observed reduction of GFAP immunoreactivity raises the question whether in the brains of FGF- $2^{-1-} / \mathrm{FGF}-5^{-1-}$ single and double mutant mice overall astrocyte numbers were reduced or whether only astrocyte differentiation was disturbed with otherwise unchanged astrocyte numbers. To answer this question, expression of the alternative astroglial marker protein S100 was

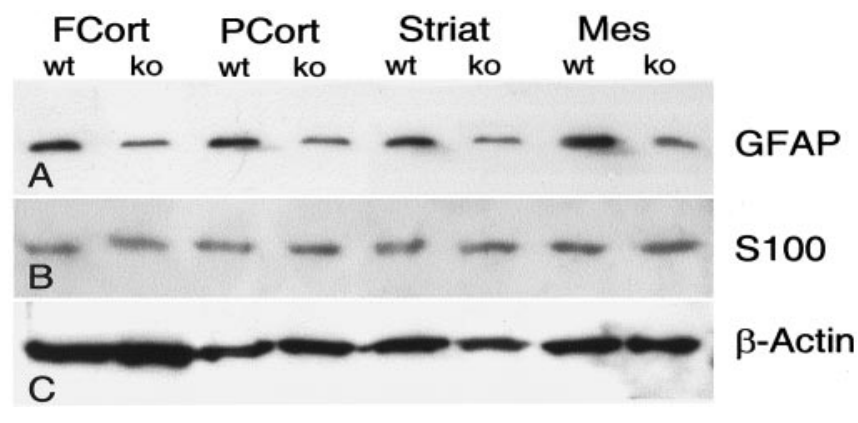

Figure 4. Western blot analysis of the astroglial marker proteins GFAP and S100 in different brain regions of FGF-2 $2^{-1-} / \mathrm{FGF}-5^{-/-}$double mutant mice and wild-type littermates. GFAP immunoreactivity is clearly decreased in all brain areas studied: frontal cortex (FCort), parietal cortex (PCort), striatum (Striat), and mesencephalon (Mes); note that levels of S100 are not overtly affected.

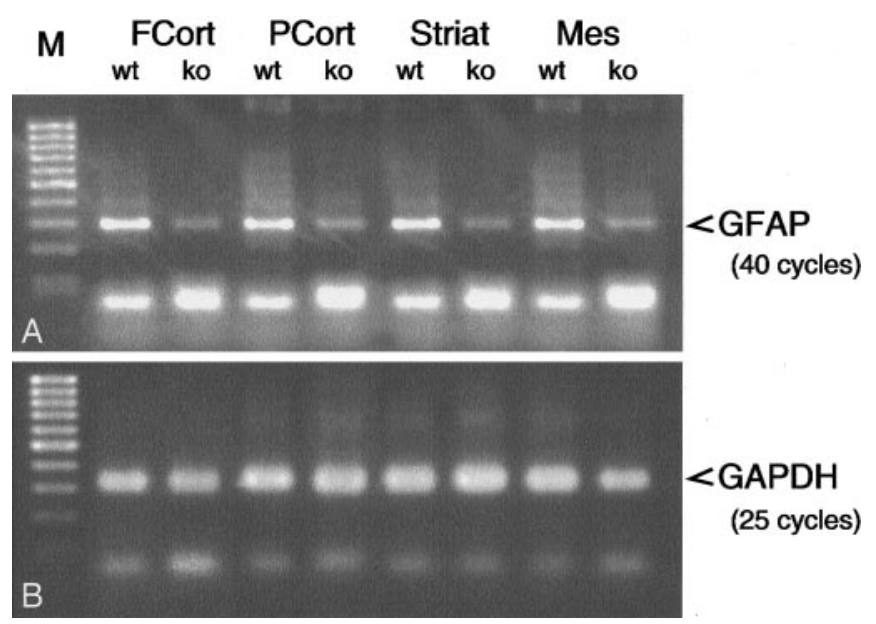

Figure 5. GFAP mRNA expression as revealed by semiquantitative RT-PCR. A, PCR products for GFAP derived from whole-cell RNA of frontal cortex (FCort), parietal cortex (PCort), striatum (Striat), and mesencephalon (Mes) of FGF- $2^{-1-} /$ FGF- $5^{-1-}$ double mutant mice are clearly reduced as compared with wild-type littermates. $B$, Parallel amplification of the housekeeping gene GAPDH revealed no apparent differences in the intensities of $P C R$ products.

analyzed immunohistochemically, and numbers of S100-positive cells were counted in the same brain regions as for the analysis of expression of GFAP. As shown in Figure 3, $C$ and $D$, immunoreactivity for $\mathrm{S} 100$ could be detected in the frontal cortex of wildtype and FGF- $2^{-1-} / \mathrm{FGF}-5^{-1-}$ double mutant mice irrespective of presence or absence of GFAP immunoreactivity (Fig. 3, compare $A, B)$. The quantitative evaluation of S100-positive cells in frontal cortex, corpus callosum, striatum, and midbrain tegmentum of wild-type and FGF- $2^{-1-} / \mathrm{FGF}-5^{-1-}$ double mutant mice (Fig. 3E) revealed no significant differences between knock-out mice and wild-type littermates.

This could be confirmed by Western blot analysis of GFAP and S100 immunoreactivity in different brain regions of wildtype and FGF- $2^{-1-} / \mathrm{FGF}-5^{-1-}$ double KO mice. Whereas GFAP immunoreactivity (Fig. $4 A$ ) was reduced in frontal cortex, parietal cortex, striatum, and mesencephalon of FGF-2 ${ }^{-1-} /$ FGF$5^{-1-}$ double mutant mice as compared with wild-type littermates, immunoreactivity for $\mathrm{S} 100$ (Fig. $4 B$ ) remained constant. Levels of $\beta$-actin were not overtly changed (Fig. $4 C$ ).

As shown on Figure $5 A$, reduced levels of GFAP in the brains of FGF-2 ${ }^{-1-} /$ FGF- $^{-/-}$double mutant mice could also be observed on the mRNA level, as revealed by RT-PCR. Signal intensities of the amplification products for GFAP derived from RNA 


\section{FGF-2/FGF-5}

wt
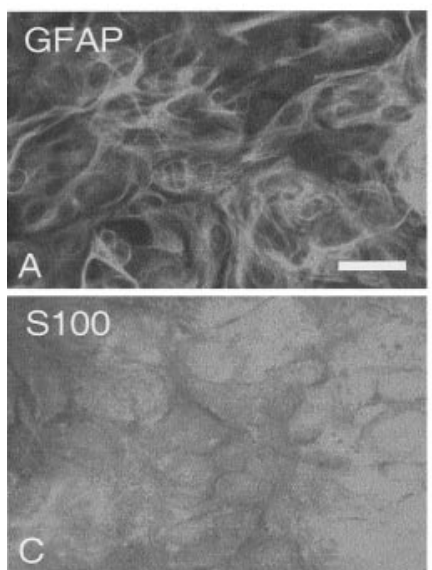

wt ko

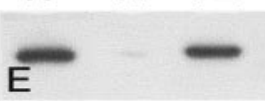

F5 F2/F5

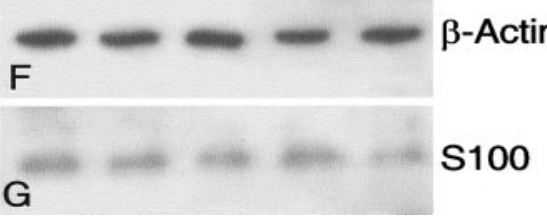

Figure 6. $\operatorname{GFAP}(A, B)$ and $\mathrm{S} 100(C, D)$ immunoreactivities in primary cultures of cortical astrocytes from FGF-2 $2^{-1-} / \mathrm{FGF}-5^{-1-}$ double mutant mice $(B, D)$ and wild-type littermates $(A$, C). Note reduction of GFAP immunoreactivity in cultures established from knock-out mice, with no apparent reduction in $\$ 100$ immunoreactivity. $E-G$, Reduced levels of GFAP revealed by Western blot analysis can be rescued by application of exogenous FGF-2 alone or in combination with FGF-5. Note that FGF-5 failed to rescue the GFAP phenotype in cortical astrocytes (compare Fig. 2). Scale bar, $20 \mu \mathrm{m}$.

samples of frontal cortex, parietal cortex, striatum, and midbrain tegmentum of FGF-2 ${ }^{-/-} / \mathrm{FGF}-5^{-/-}$double mutant mice were greatly reduced as compared with those from wild-type littermates. In contrast, amplification products for the housekeeping gene GAPDH, derived from the same RNA samples revealed no changes in the knock-outs as compared with wild-type controls (Fig. 5B).

\section{Reduced levels of GFAP in cortical astrocytes of FGF-2 $-1-$,} FGF-5 ${ }^{-1-}$ double mutant mice can be rescued by exogenously applied FGF-2, but not FGF-5

To substantiate the specific role of FGF-2 for the expression of GFAP in cortical astrocytes in vivo, we established primary astroglial cultures from the cortex of neonatal wild-type and FGF-2 ${ }^{-1-} / \mathrm{FGF}-5^{-1-}$ double mutant mice. Cortical astrocytes from wild-type mice revealed abundant expression of GFAP (Fig. $6 A)$. Sister cultures established from FGF-2 $-1-/$ FGF-5 $-1-$ double-deficient mice revealed that only very few cells were immunopositive for GFAP (Fig. $6 \mathrm{~B}$ ). In contrast, an alternative astroglial marker, $\mathrm{S} 100$, was apparently not affected (Fig. 6C,D). To further substantiate the notion that losses of GFAP in cortical astrocytes seen in FGF-2 ${ }^{-/-} / \mathrm{FGF}-5^{-/-}$double mutant mice are attributable to the lack of FGF-2 rather than FGF-5, cultures of cortical astroglial cultures of FGF-2 ${ }^{-/-} / \mathrm{FGF}-5^{-/-}$double mutant mice were treated with FGF-2 or FGF-5, respectively, and were subsequently analyzed for GFAP immunoreactivity using Western blotting. As shown in Figure 6E, immunoreactivity for GFAP was drastically reduced in samples from cortical astroglial cultures of FGF-2 $2^{-/-} / \mathrm{FGF}-5^{-/-}$double-deficient mice, as compared with cultures from wild-type littermates. The loss in GFAP was fully reversed by exogenously applied FGF-2 but not FGF-5 (10 ng/ml each) (Fig. $6 E$ ). Levels of $\beta$-actin and of $S 100$ were not changed (Fig. 6F,G).

\section{Intermediate filament density is reduced in perivascular astroglial endfeet of FGF-2 ${ }^{-1-} /$ FGF-5 $5^{-/-}$double mutant mice}

Astrocytes enwrap brain capillaries by a continuous layer of their perivascular endfeet. We therefore investigated whether the observed reduction of GFAP in FGF- $2^{-1-} / \mathrm{FGF}-5^{-1-}$ double mutant mice implied structural changes in astroglial perivascular endfeet. Immunocytochemistry revealed that GFAP immunoreactivity was indeed reduced in perivascular endfeet of striatal astroglial cells of FGF-2 ${ }^{-/-} / \mathrm{FGF}-5^{-1-}$ double mutant mice, as compared with wild-type littermates (Fig. $7 A, B$ ). Further along this line, electron microscopical analyses (Fig. $7 C, D$ ) revealed an overall reduction in numbers of intermediate filaments in perivascular endfeet of FGF-2 ${ }^{-/-} / \mathrm{FGF}-5^{-/-}$double mutant mice (Fig. 7C), as compared with wild-type littermates (Fig. 7D). Since the observed effects did not occur in an all-or-none manner, five categories of perivascular astroglial endfeet with different grades of loss of intermediate filaments were established and proportions of endfeet with a certain degree of reduction of intermediate filament density were determined. As shown in Figure $7 E$, a shift toward categories with less or lacking intermediate filaments was found in FGF-2 ${ }^{-/-} / \mathrm{FGF}-5^{-/-}$double mutant mice, as compared with wild-type littermates.

\section{Albumin extravasation is increased and expression of tight junction proteins is decreased in brain capillaries of FGF-2 ${ }^{-/}$/FGF-5 ${ }^{-/-}$double mutant mice}

To further elucidate functional consequences of altered cytoskeletal architecture in perivascular astroglial endfeet, permeability of the BBB was assayed by immunohistochemical detection of albumin extravasation into the brain parenchyma. As demonstrated in Figure 8, albumin immunofluorescence could not be detected in wild-type mice within areas surrounding brain capillaries (Fig. $8 A$ ). In FGF-2 ${ }^{-/-} /$FGF-5 ${ }^{-/-}$double-deficient animals, however (Fig. $8 B$ ), a majority of brain capillaries showed a halo of albumin extravasation into the surrounding parenchyma, suggesting increased permeability of the BBB. To quantify this, numbers of brain capillaries with and without apparent albumin extravasation were counted in different brain regions of FGF-2 ${ }^{-1-}$, FGF-5 ${ }^{-1-}$ double mutant mice and in wild-type littermates. As demonstrated in Figure $8 C$, the proportion of brain capillaries with albumin extravasation was significantly increased in frontal cortex, striatum, and midbrain tegmentum of FGF- $2^{-/-} / \mathrm{FGF}-5^{-/-}$double mutant mice, as compared with wild-type littermates. In contrast, in the corpus callosum, no significant changes in albumin extravasation in FGF-2 ${ }^{-/-} /$FGF-5 ${ }^{-/-}$double mutant mice was observed, as compared with their wild-type littermates.

To clarify the molecular basis for the observed increase in BBB permeability, expression of the tight junction proteins ZO-1 and Occludin was analyzed in striatal brain capillaries by indirect immunofluorescence and by Western blotting. Figure $9 A$ reveals a distinct expression pattern of ZO-1 in capillaries of the wildtype striatum, whereas ZO-1 immunoreactivity was clearly reduced in striatal capillaries of FGF- $2^{-1-} / \mathrm{FGF}-5^{-/-}$double mu- 


\section{FGF-2/FGF-5}

wt
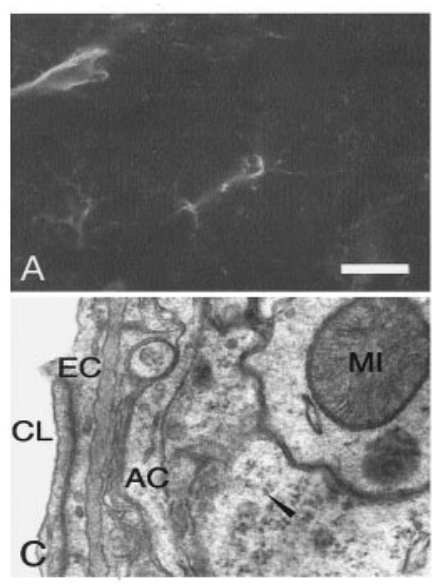
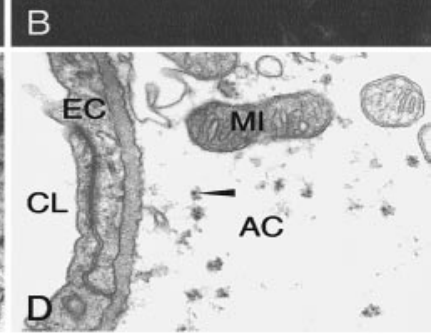

Categories of intermediate filament density in perivascular astroglial endfeet: Cat I = normal density Cat II = slightly reduced density Cat III = reduced density

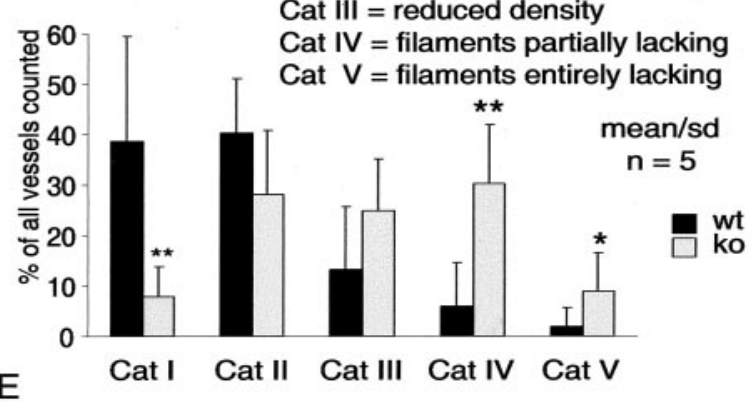

Figure 7. $A, B$, Reduced levels of GFAP immunoreactivity in perivascular astroglial endfeet in the striata of FGF-2/FGF-5 double mutant mice $(B)$ as compared with wild-type littermates $(A)$. Scale bar, $20 \mu \mathrm{m}$. C, D, Electron microscopic images of representative areas showing capillary endothelial cells (EC) with adjacent basal lamina $\left({ }^{*}\right)$ and astroglial endfeet (AC). Note the reduced number of intermediate filaments (arrowhead) and a disrupted cytoskeleton in the astroglial endfoot in the striatum of an FGF-2/FGF-5 double mutant mouse. MI, Mitochondrion. Scale bar, $1 \mu \mathrm{m}$. E, Frequency distribution of five distinct categories (Cat I-V) of perivascular astroglial endfeet distinguished by the density of intermediate filaments, as depicted in Cand $D$. Comparison of FGF-2/FGF-5 double mutant mice and wild-type littermates: 100 capillaries were evaluated for each of five wild-type and knock-out mice. Data are given as the mean percentage of all vessels evaluated. ${ }^{*} p<0.05 ;{ }^{* *} p<0.01$.

tant mice (Fig. 9B). In addition, Occludin immunoreactivity, which is abundant in capillaries of wild-type animals, was similarly reduced in capillaries of FGF- $2^{-/-} / \mathrm{FGF}-5^{-/-}$mutant mice (Fig. 9C,D). This could be confirmed by Western blot analysis, showing a distinct reduction of Occludin immunoreactivity in frontal and parietal cortex, striatum, and mesencephalon of FGF-2 ${ }^{-1-} /$ FGF-5 ${ }^{-1-}$ double mutant mice, as compared with wild-type littermates (Fig. 9E). Together, these results suggest that alterations in GFAP intermediate filaments caused by FGF-2 and FGF-5 deficiencies are accompanied by alterations in BBB permeability and expression of essential tight junction proteins.

\section{Discussion}

The present analysis of mice lacking FGF-2 or FGF-5 demonstrates for the first time that endogenous production of these factors is crucial for regulating differentiation of gray matter astrocytes in a region-specific manner. Cortical and striatal astrocytes of mouse mutants lacking FGF-2 show substantial losses of

\section{FGF-2/FGF-5}

wt

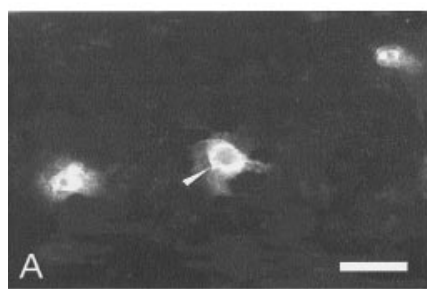

C

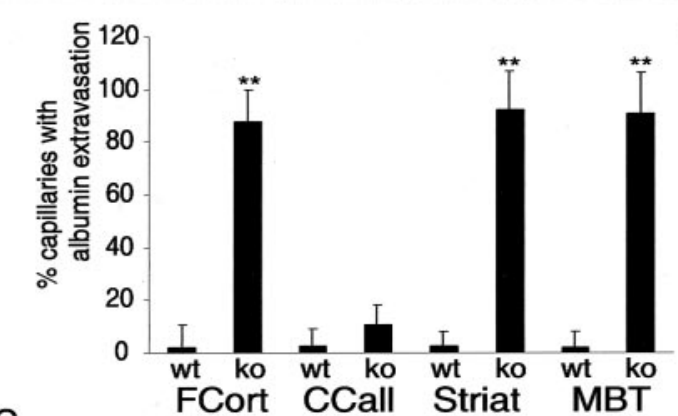

ko

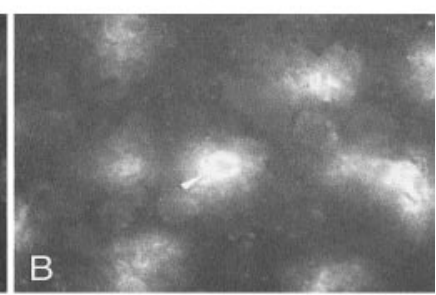

mean/sd

Figure 8. Increased albumin extravasation in the striatum of FGF-2 $2^{-/-} / \mathrm{FGF}-5^{-/-}$double mutant mice $(B)$ as compared with wild-type littermates $(A)$. Note that the strong albuminspecific immunoreactivity in the parenchyma adjacent to striatal blood vessels (arrowheads) in FGF- $2^{-1-} / \mathrm{FGF}-5^{-1-}$ double mutant mice $(B)$ is lacking in wild-type littermates $(A)$. Scale bar, $20 \mu \mathrm{m}$. C, Statistical evaluation of the proportion of brain capillaries with apparent albumin extravasation in frontal cortex (FCort), corpus callosum (CCall), striatum (Striat), and midbrain tegmentum (MBT) of FGF-2 $2^{-1-} / \mathrm{FGF}-5^{-1-}$ double mutant mice (ko) and their wild-type littermates (wt). Seven consecutive sections with a section interval of $50 \mu \mathrm{m}$ were evaluated for each of six wild-type and knock-out animals.

the astroglial intermediate filament protein GFAP and its mRNA, whereas FGF-5-deficient mice display a similar defect in the midbrain tegmentum. In contrast, white matter astrocytes in the corpus callosum were not affected by the loss of FGF-2 or FGF-5. FGF-2/FGF-5 double mutants did not show additional or more drastic phenotypes, suggesting no or negligible synergies of FGF-2 and FGF-5 in the regulation of astroglial differentiation. The alternative astroglial marker protein S100 was not affected in FGF-2 ${ }^{-/-} /$FGF-5 ${ }^{-/}$double mutant mice, suggesting disturbed astrocyte differentiation rather than astrocyte loss to underlie the observed effects. Reduced levels of GFAP were also observed in perivascular astroglial endfeet, and this phenotype was accompanied by a leaky BBB, as detected by albumin extravasation from brain capillaries and reduced levels of the tight junction proteins ZO-1 and Occludin in endothelial cells of brain capillaries.

Induction of astroglial GFAP expression by FGF-2 has been demonstrated in many studies either by application of exogenous FGF-2 in vivo (Eclancher et al., 1990; Otto and Unsicker, 1994) or by treatment of primary astroglial cultures in vitro (Perraud et al., 1988; Yoshida and Gage, 1991; Petroski et al., 1991; Otto and Unsicker, 1993). It is also well established that upregulation of endogenous FGF-2 after traumatic and ischemic insults results in increased GFAP expression (Kiyota et al., 1991; Takami et al., 1993). Even so, a physiological role of FGF-2 in the unlesioned CNS had not been established as yet. Moreover, putative functions of FGF-5 in the regulation of astroglial cell differentiation had previously been addressed only with regard to gap junction formation (Reuss et al., 2000a).

Astrocytes consist of a variety of subpopulations with high regional specificity concerning their morphologies, gene expres- 


\section{FGF-2}

wt
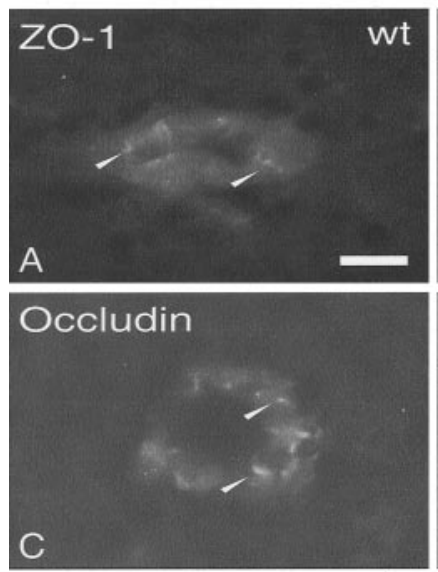

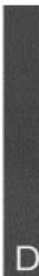

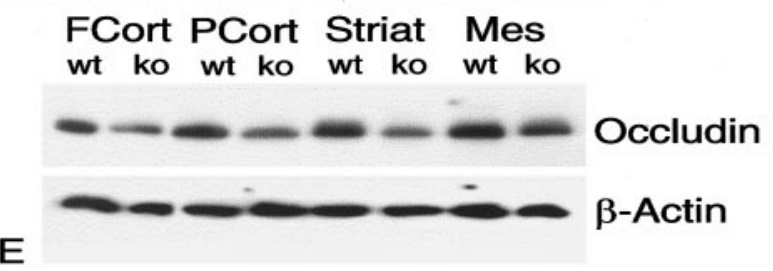

Figure 9. Immunofluorescence showing decreased levels of tight junction proteins $20-1$ and 0 ccludin (arrowheads) in striatal blood vessels of FGF- $2^{-1-} / \mathrm{FGF}-5^{-1-}$ double mutant mice $(B, D)$ as compared with wild-type littermates $(A, C)$. Scale bar, $10 \mu \mathrm{m}$. $E$, Western blot analysis revealed decreased levels of 0 ccludin in all brain areas studied. FCort, Frontal cortex; PCort, parietal cortex; Striat, striatum; Mes, mesencephalon. Scale bar, $10 \mu \mathrm{m}$.

sion patterns, and gene regulation, and even distinct progenitor cells (for review, see Wilkin et al., 1990). Most, but not all astroglial subtypes seem to share an expression of GFAP (Eng, 1985; Eng and Ghirnikar, 1994), vimentin (Tapscott et al., 1981), and glutamine synthetase (Martinez-Hernandez et al., 1977). Different morphological subtypes, designated as "protoplasmic" and "fibrillar" astrocytes, have long been known and may correspond to differences in GFAP expression (Eng, 1985). Type I and type II astrocytes have been distinguished in cultures established from the optic nerve based on the expression of the A2B5 antigen and distinct patterns of ion channels (Raff et al., 1983; Minturn et al., 1990). Distinct sets of neurotransmitter receptors and transporters are expressed by astrocytes in defined regions of the CNS. For example, Bergmann glial cells in the cerebellum express functional receptors for glutamate, GABA, and histamine, but not for glycine and dopamine (Kirischuk et al., 1996; Müller and Kettenmann, 1995). Glycine receptors instead are found in astroglial cells of the spinal cord, a CNS region with prominent glycinergic transmission. Dopamine receptors are abundant in astrocytes of the striatum (Reuss et al., 2000b), consistent with its rich dopaminergic innervation. Together, there is ample evidence to suggest that a region-specific expression of astroglial transmitter receptors and transporters may reflect regional differences in predominant neuronal transmitter phenotypes. Astroglial heterogeneity also includes connexin function and expression levels. Thus, Batter et al., (1992) have shown that connexin 43 expression and functional coupling is higher in rat hypothalamic than striatal astrocytes. Moreover, we have previously shown that FGF-2, FGF-5, and FGF-9 regulate connexin 43 expression and coupling in cultured astroglia from cortex, striatum, and midbrain in a region-specific manner (Reuss et al., 1998, 2000a).
The present study establishes a link between FGF-2- and FGF5 -dependent induction of GFAP to features of astroglial cells that are subject to a regionally distinct regulation. Interestingly, region specificities included astroglial GFAP both in different brain areas and in gray versus white matter astrocytes. A possible explanation for these differences might lie in distinct expression patterns of FGFRs. A previous study had failed to reveal regionspecific differences in astroglial FGFR in the rat (Reuss et al., 2000a). Region-specific expression of FGFR has been described during development of the chick brain (Wilke et al., 1997; Walshe and Mason, 2000), where FGFR-2 and FGFR-3 are expressed in the diencephalon and metencephalon at Hamburger-Hamilton ( $\mathrm{HH}$ ) stages 9-11, whereas at stage HH 21 FGFR-2 is expressed in telencephalon, mesencephalon, and metencephalon, and FGFR-3 is expressed in diencephalon and metencephalon. However, the situation is getting even more complicated, considering the fact that receptor-binding specificity of FGFR is mainly determined by alternative splicing of their third Ig-like domains (Werner et al., 1992; Wang et al., 1995; Ornitz et al., 1996).

GFAP is post-translationally modified by phosphorylation, a process that is involved in the regulation of assembly and disassembly of astroglial intermediate filament proteins (for review, see Inagaki et al., 1994). Because it is well established that protein phosphorylation has a major influence on protein conformation and thereby on antibody binding, this raises the question, whether differences in GFAP immunoreactivity seen in wild-type and FGF mutant mice might be a result of different phosphorylation patterns rather than protein levels. This is unlikely, because levels of GFAP mRNA as revealed by RT-PCR were also reduced. Hence, changes in GFAP are probably caused by altered transcription or translation rather than post-translational modifications.

Besides direct actions of FGF-2 and FGF- 5 on astroglial GFAP expression, also indirect mechanisms of GFAP induction are conceivable. Several growth factors, as e.g., NGF, TGF- $\beta 1$, and GDNF, have been shown to be upregulated by FGF-2 in reactive astroglial cells (Yoshida and Gage, 1991; Suter-Crazzolara and Unsicker, 1996; Krieglstein et al., 1998), but up to now none of them has been shown to influence GFAP synthesis.

To address the issue whether the reduction of GFAP expression in FGF-deficient mice resulted from a loss of astrocytes or a decrease in GFAP expression, we used an alternative astroglial marker, S100. Our data suggest that FGF-2 and FGF-5 deficiencies specifically affect GFAP expression, leaving numbers of astrocytes unchanged. This notion is corroborated by electron microscopy, which clearly demonstrated the presence of perivascular astroglial endfeet in FGF-deficient mice. Independent regulation of GFAP and S100 have been also described in the visual cortex of the marmoset (Missler et al., 1994), in which GFAP is transiently downregulated during postnatal development, whereas S100 steadily increases after birth. Likewise, treatment with glucocorticoids leads to reduced expression of GFAP but not of S100 in the rat cortex and hippocampus (O'Callaghan et al., 1991).

The drastic decrease in GFAP expression seen in FGF mutant mice could be rescued by treating cortical astrocytes derived from such brains with FGF-2. This suggests that, whatever additional biochemical alterations may occur in FGF-deprived astrocytes, as e.g., changes in receptors, signaling components, or other growth factors, FGF-2 alone is sufficient to overcome the functional consequences of the FGF-2 knock-out with regard to GFAP deficiency.

As the present study demonstrates, reduced levels of GFAP in 
perivascular astroglial endfeet is paralleled by decreased densities of intermediate filaments, finally resulting in increased permeability of the blood-brain barrier. A similar phenomenon has been previously demonstrated for mice with a gene defect for GFAP, astrocytes of which failed to induce normal transendothelial resistance in cultured brain endothelial cells (Pekny et al., 1998). Transendothelial resistance is a direct measure for tight junction formation (Krause et al., 1991; Biegel et al., 1995). Further along this line, reduced levels of the tight junction proteins ZO-1 and Occludin and extravasation of albumin from brain capillaries found in our study also reflect a functional deficit in the BBB that may directly depend on the deficit of GFAP in perivascular astroglial endfeet. Alternatively, FGF-2 and FGF-5 may be conceived to regulate endothelial tight junctions directly, as it has been shown for the expression of ZO-1 in the rat cornea endothelium (Petroll et al., 1996).

The prominent and region-specific losses of GFAP seen in FGF-2 and FGF-5 mutant mice might have additional consequences for brain functions. Interactions of astroglial processes with synapses in the supraoptic and paraventricular nuclei of the hypothalamus are subject to plastic changes responding to parturition or dehydration (Hatton et al., 1984; Perlmutter et al., 1984). Another example of such plasticity are synapses on facial motoneurons that are stripped from the cell bodies by astroglial processes (Jones et al., 1997). Further along this line, deficits in long-term potentiation or depression using cerebellar and hippocampal learning paradigms have been revealed in GFAP knock-out mice (McCall et al., 1996; Shibuki et al., 1996). Similar deficits may be conceivable for FGF knock-out mice.

In conclusion, the present results reveal an important physiological role of endogenously synthesized FGF-2 and FGF-5 in the regulation of astroglial differentiation and $\mathrm{BBB}$ integrity. These findings open new avenues for further analysis of the functional consequences of disturbed FGF synthesis and their effects on the astroglial cytoskeletal network for synaptic plasticity and repair under pathological circumstances.

\section{References}

Batter DK, Corpina RA, Roy C, Spray DC, Hertzberg EL, Kessler JA (1992) Heterogeneity in gap junction expression in astrocytes cultured from different brain regions. Glia 6:213-221.

Biegel D, Spencer DD, Pachter JS (1995) Isolation and culture of human brain microvessel endothelial cells for the study of blood-brain barrier properties in vitro. Brain Res 692:183-189.

Bieger S, Unsicker K (1996) Functions of fibroblast growth factors (FGFs) in the nervous system. In: Chemical factors in neural growth, degeneration and repair (Bell C, ed), pp 339-375. Amsterdam: Elsevier.

Bignami A, Eng LF, Dahl D, Uyeda CT (1972) Localization of the glial fibrillary acidic protein in astrocytes by immunofluorescence. Brain Res 43:429-435.

Dono R, Texido G, Dussel R, Ehmke H, Zeller R (1998) Impaired cerebral cortex development and blood pressure regulation in FGF-2-deficient mice. EMBO J 17:4213-4225.

Eclancher F, Perraud F, Faltin J, Labourdette G, Sensenbrenner M (1990) Reactive astrogliosis after basic fibroblast growth factor (bFGF) injection in injured neonatal rat brain. Glia 3:502-509.

Eclancher F, Kehrli P, Labourdette G, Sensenbrenner M (1996) Basic fibroblast growth factor (bFGF) injection activates the glial reaction in the injured adult rat brain. Brain Res 737:201-214.

Eng LF (1985) Glial fibrillary acidic protein (GFAP): the major protein of glial intermediate filaments in differentiated astrocytes. J Neuroimmunol 8:203-214.

Eng LF, Ghirnikar RS (1994) GFAP and astrogliosis. Brain Pathol 4:229-237.

Eng LF, Vanderhaeghen JJ, Bignami A, Gerstl B (1971) An acidic protein isolated from fibrous astrocytes. Brain Res 28:345-351.
Frautschy SA, Walicke PA, Baird A (1991) Localization of basic fibroblast growth factor and its mRNA after CNS injury. Brain Res 553:291-299.

Gomez-Pinilla F, Cotman CW (1993) Distribution of fibroblast growth factor 5 mRNA in the rat brain: an in situ hybridization study. Brain Res 606:79-86.

Gomez-Pinilla F, Lee JW, Cotman CW (1994) Distribution of basic fibroblast growth factor in the developing rat brain. Neuroscience 61:911-923.

Hatton GI, Perlmutter LS, Salm AK, Tweedle CD (1984) Dynamic neuronal-glial interactions in hypothalamus and pituitary: implications for control of hormone synthesis and release. Peptides 5 (S1):121-138.

Haub O, Drucker B, Goldfarb M (1990) Expression of the murine fibroblast growth factor 5 gene in the adult central nervous system. Proc Natl Acad Sci USA 87:8022-8026.

Hébert JM, Rosenquist T, Gotz J, Martin GR (1994) FGF5 as a regulator of the hair growth cycle: evidence from targeted and spontaneous mutations. Cell 78:1017-1025.

Henkel AW, Bieger SC (1994) Quantification of proteins dissolved in an electrophoresis sample buffer. Anal Biochem 223:329-331.

Inagaki M, Nakamura Y, Takeda M, Nishimura T, Inagaki N (1994) Glial fibrillary acidic protein: dynamic property and regulation by phosphorylation. Brain Pathol 4:239-243.

Jones LL, Kreutzberg GW, Raivich G (1997) Regulation of CD44 in the regenerating mouse facial motor nucleus. Eur J Neurosci 9:1854-1863.

Kirischuk S, Tuschick S, Verkhratsky A, Kettenmann H (1996) Calcium signalling in mouse Bergmann glial cells mediated by alpha1adrenoreceptors and $\mathrm{H} 1$ histamine receptors. Eur J Neurosci 8:1198-1208.

Kiyota Y, Takami K, Iwane M, Shino A, Miyamoto M, Tsukuda R, Nagaoka A (1991) Increase in basic fibroblast growth factor-like immunoreactivity in rat brain after forebrain ischemia. Brain Res 545:322-328.

Krause D, Mischeck U, Galla HJ, Dermietzel R (1991) Correlation of zonula occludens ZO-1 antigen expression and transendothelial resistance in porcine and rat cultured cerebral endothelial cells. Neurosci Lett 128:301-304.

Krieglstein K, Reuss B, Maysinger D, Unsicker K (1998) Short communication: transforming growth factor-beta mediates the neurotrophic effect of fibroblast growth factor-2 on midbrain dopaminergic neurons. Eur J Neurosci 10:2746-2750.

Laemmli UK (1970) Cleavage of structural proteins during the assembly of the head of bacteriophage T4. Nature 227:680-685.

Lindholm D, Harikka J, da Penha Berzaghi M, Castren E, Tzimagiorgis G, Hughes RA, Thoenen H (1994) Fibroblast growth factor-5 promotes differentiation of cultured rat septal cholinergic and raphe serotonergic neurons: comparison with the effects of neurotrophins. Eur J Neurosci 6:244-252.

Luft JH (1961) Improvements in epoxy resin embedding methods. J Biophys Biochem Cytol 9:409-414.

Martinez-Hernandez A, Bell KP, Norenberg MD (1977) Glutamine synthetase: glial localization in brain. Science 195:1356-1358.

McCall MA, Gregg RG, Behringer RR, Brenner M, Delaney CL, Galbreath EJ, Zhang CL, Pearce RA, Chiu SY, Messing A (1996) Targeted deletion in astrocyte intermediate filament (GFAP) alters neuronal physiology. Proc Natl Acad Sci USA 93:6361-6366.

Minturn JE, Black JA, Angelides KJ, Waxman SG (1990) Sodium channel expression detected with antibody 7493 in A2B5+ and A2B5- astrocytes from rat optic nerve in vitro. Glia 3:358-367.

Missler M, Eins S, Bottcher H, Wolff JR (1994) Postnatal development of glial fibrillary acidic protein, vimentin and $\mathrm{S} 100$ protein in monkey visual cortex: evidence for a transient reduction of GFAP immunoreactivity. Brain Res Dev Brain Res 82:103-117.

Müller T, Kettenmann H (1995) Physiology of Bergmann glial cells. Int Rev Neurobiol 38:341-359.

O'Callaghan JP, Brinton RE, McEwen BS (1991) Glucocorticoids regulate the synthesis of glial fibrillary acidic protein in intact and adrenalectomized rats but do not affect its expression following brain injury. J Neurochem $57: 860-869$.

Ornitz DM, Xu J, Colvin JS, McEwen DG, MacArthur CA, Coulier F, Gao G, Goldfarb M (1996) Receptor specificity of the fibroblast growth factor family. J Biol Chem 271:15292-15297.

Otto D, Unsicker K (1993) FGF-2-mediated protection of cultured mesencephalic dopaminergic neurons against MPTP and $\mathrm{MPP}^{+}$: specificity and 
impact of culture conditions, non-dopaminergic neurons, and astroglial cells. J Neurosci Res 34:382-393.

Otto D, Unsicker K (1994) FGF-2 in the MPTP model of Parkinson's disease: effects on astroglial cells. Glia 11:47-56.

Ozawa K, Uruno T, Miyakawa K, Seo M, Imamura T (1996) Expression of the fibroblast growth factor family and their receptor family genes during mouse brain development. Brain Res Mol Brain Res 41:279-288.

Pekny M, Stanness KA, Eliasson C, Betsholtz C, Janigro D (1998) Impaired induction of blood-brain barrier properties in aortic endothelial cells by astrocytes from GFAP-deficient mice. Glia 22:390-400.

Perlmutter LS, Tweedle CD, Hatton GI (1984) Neuronal/glial plasticity in the supraoptic dendritic zone: dendritic bundling and double synapseformation at parturition. Neuroscience 13:769-779.

Perraud F, Labourdette G, Miehe M, Loret C, Sensenbrenner M (1988) Comparison of the morphological effects of acidic and basic fibroblast growth factors on rat astroblasts in culture. J Neurosci Res 20:1-11.

Perraud F, Labourdette G, Eclancher F, Sensenbrenner M (1990) Primary cultures of astrocytes from different brain areas of newborn rats and effects of basic fibroblast growth factor. Dev Neurosci 12:11-21.

Petroll WM, Jester JV, Barry-Lane PA, Cavanagh HD (1996) Effects of basic FGF and TGF b1 on F-actin and ZO-1 organization during cat endothelial wound healing. Cornea 15:525-532.

Petroski RE, Grierson JP, Choi-Kwon S, Geller HM (1991) Basic fibroblast growth factor regulates the ability of astrocytes to support hypothalamic neuronal survival in vitro. Dev Biol 147:1-13.

Prat A, Biernacki K, Wosik K, Antel J (2001) Glial cell influence on the human blood-brain barrier. Glia 36:145-155.

Raff MC, Abney ER, Cohen J, Lindsay R, Noble M (1983) Two types of astrocytes in cultures of developing rat white matter: differences in morphology, surface gangliosides, and growth characteristics. J Neurosci 3:1289-1300

Ransom BR, Kettenmann H (1995) Neuroglia. Oxford: Oxford UP.

Reuss B, Dermietzel R, Unsicker K (1998) Fibroblast growth factor 2 (FGF-2) differentially regulates connexin (cx) 43 expression and function in astroglial cells from distinct brain regions. Glia 22:19-30.

Reuss B, Hertel M, Werner S, Unsicker K (2000a) Fibroblast growth factors-5 and -9 distinctly regulate expression and function of the gap junction protein connexin 43 in cultured astroglial cells from different brain regions. Glia 30:231-241.

Reuss B, Leung D, Ohlemeyer C, Kettenmann H, Unsicker K (2000b) Re- gionally distinct regulation of astroglial neurotransmitter receptors by fibroblast growth factor-2. Mol Cell Neurosci 16:42-58.

Reynolds ES (1963) The use of lead citrate at high pH as an electron-opaque stain in electron microscopy. J Cell Biol 17:208-212.

Shibuki K, Gomi H, Chen L, Bao S, Kim JJ, Wakatsuki H, Fujisaki T, Fujimoto K, Katoh A, Ikeda T, Chen C, Thompson RF, Itohara S (1996) Deficient cerebellar long-term depression, impaired eyeblink conditioning, and normal motor coordination in GFAP-mutant mice. Neuron 16:587-599.

Suter-Crazzolara C, Unsicker K (1996) GDNF mRNA levels are induced by FGF-2 in rat C6 glioblastoma cells. Brain Res Mol Brain Res 41:175-182.

Takami K, Kiyota Y, Iwane M, Miyamoto M, Tsukuda R, Igarashi K, Shino A, Wanaka A, Shiosaka S, Tohyama M (1993) Upregulation of fibroblast growth factor-receptor messenger RNA expression in rat brain following transient forebrain ischemia. Exp Brain Res 97:185-194.

Tapscott SJ, Bennett GS, Toyama Y, Kleinbart F, Holtzer H (1981) Intermediate filament proteins in the developing chick spinal cord. Dev Biol $86: 40-54$.

Towbin H, Staehelin T, Gordon J (1979) Electrophoretic transfer of proteins from polyacrylamide gels to nitrocellulose sheets: procedure and some applications. Proc Natl Acad Sci USA 76:4350-4354.

Walshe J, Mason L (2000) Expression of FGFR1, FGFR2, and FGFR3 during early neural development in the chick embryo. Mech Dev 90:103-110.

Wang F, Kan M, Xu J, Yan G, McKeehan WL (1995) Ligand-specific structural domains in the fibroblast growth factor receptor. J Biol Chem 270:10222-10230.

Werner S, Duan DS, de Vries C, Peters KG, Johnson DE, Williams LT (1992) Differential splicing in the extracellular region of fibroblast growth factor receptor 1 generates receptor variants with different ligand-binding specificities. Mol Cell Biol 12:82-88.

Wilke TA, Gubbles S, Schwartz J, Richman JM (1997) Expression of fibroblast growth factor receptors (FGFR1, FGFR2, FGFR3) in the developing head and face. Dev Dyn 210:41-52.

Wilkin GP, Marriott DR, Cholewinski AJ (1990) Astrocyte heterogeneity. Trends Neurosci 13:43-46.

Yoshida K, Gage FH (1991) Fibroblast growth factors stimulate nerve growth factor synthesis and secretion by astrocytes. Brain Res 538: $118-126$.

Zhou M, Sutliff RL, Paul RJ, Lorenz JN, Hoying JB, Haudenschild CC, Yin M, Coffin JD, Kong L, Kranias EG, Luo W, Boivin GP, Duffy JJ, Pawlowski SA, Doetschman T (1998) Fibroblast growth factor 2 control of vascular tone. Nat Med 4:201-207. 